\title{
MANIFOLDS WITH MANY COMPLEX STRUCTURES
}

\author{
By DOMINIC D. JOYCE
}

published as Quart. J. Math. Oxford 46 (1995), 169-184.

\section{Introduction}

Let $G$ be a Lie subgroup of $G L(n)$. The bundle of frames $F$ on a manifold $M^{n}$ is a principal $G L(n)$ - bundle over $M$, and a $G$-structure on $M$ is a principal subbundle $P$ of $F$ with group $G$, using the given inclusion $G \subset G L(n)$ and not some other. By a geometric structure on $M$ we mean a $G$ - structure on $M$ for some Lie subgroup $G$ of $G L(n)$, that satisfies an integrability condition, which must be a (possibly trivial) partial differential equation involving only the $G$ - structure. Here are some examples of geometric structures:

- The Riemannian metric has $G=O(n)$, and trivial integrability condition. Some nontrivial integrability conditions are for the metric to have constant scalar curvature or to be Ricci-flat.

- Orientation is a geometric structure with $G=G L_{+}(n)$, the matrices of positive determinant, and trivial integrability condition.

- The complex structure has $G=G L(n, \mathbb{C})$, and integrability condition the vanishing of the Nijenhuis tensor.

A geometric structure is said to be trivial if it is locally isomorphic to the standard model on $\mathbb{R}^{n}$. Complex structures are always trivial in this sense. An important problem in differential geometry is to produce nontrivial examples of geometric structures that have nontrivial integrability conditions.

A hypercomplex structure $([\mathbf{1 0}$, p. 137]; [6], [7]) is a collection of three integrable complex structures $I_{1}, I_{2}, I_{3}$ satisfying $I_{1} I_{2}=I_{3}$ on a manifold $M$ of dimension $4 n$, and is a geometric structure in the above sense with $G=G L(n, \mathbb{H})$. In [6], [7] the author produced nontrivial examples of hypercomplex manifolds. This paper will use related methods to produce nontrivial examples of a large class of geometric structures composed of complex structures, defined next.

Definition 1.1. Let $B$ be a subset of $\left\{j \in G L(2 n): j^{2}=-1\right\} \subset G L(2 n)$, and $G$ be the subgroup $\{x \in G L(2 n): x j=j x$ for all $j \in B\}$ of $G L(2 n)$. Then a $G$ - structure on a manifold $M^{2 n}$ induces an almost complex structure $J$ on $M$ for every element $j$ of $B$. Define the geometric structure associated to $B$ to be the $G$ - structure, with the integrability condition that the almost complex structure induced by each member of $B$ should be integrable.

Complex and hypercomplex structures are of this form. A convenient notation for these structures uses algebras and modules. For the rest of this article, let an algebra $A$ mean a finite-dimensional algebra with 1 over $\mathbb{R}$, and an $A$ - module mean a finitedimensional, unital, left module over $A$. Also, let $M(n, A)$ denote the algebra of $n \times n$ matrices with entries in $A$. If $B \subset G L(2 n)$ is as above, define $A$ to be the subalgebra of $M(2 n, \mathbb{R})$ generated over $\mathbb{R}$ by $B$. Then $A$ is an algebra, $B \subset\left\{a \in A: a^{2}=-1\right\}$, and $\mathbb{R}^{2 n}$ is an $A$ - module in the obvious way. Conversely, if $A$ is an algebra and $B \subset\left\{a \in A: a^{2}=-1\right\}$ then any $A$ - module gives rise to a geometric structure associated to $B$. For instance, when 
$A$ is the quaternions $\mathbb{H}$ and $B=\left\{j_{1}, j_{2}, j_{3}\right\}$, the $A$ - module $\mathbb{H}^{n}$ gives the hypercomplex structure in dimension $4 n$.

An important family of algebras are the Clifford algebras, which were studied by Atiyah et al. in $[\mathbf{1}]$. Let $V=\mathbb{R}^{n}$ with the usual distance |.|, and $T_{n}$ be the infinitedimensional graded algebra $T_{n}=\bigoplus_{i=0}^{\infty} \otimes^{i} V$, where $\otimes^{0} V=\mathbb{R}$, and multiplication is by tensor products in the obvious way. Let $I_{n}$ be the two-sided ideal of $T_{n}$ generated by elements of the form $x \otimes x+|x|^{2} \cdot 1$ for $x \in V$. Define $C_{n}$ to be the quotient algebra $T_{n} / I_{n}$. Then $C_{n}$ is the $n^{\text {th }}$ Clifford algebra, as defined in $[\mathbf{1}, \S 2]$. The first three Clifford algebras are $C_{0} \cong \mathbb{R}, C_{1} \cong \mathbb{C}$ and $C_{2} \cong \mathbb{H}$, and from [1, Table 1], the sequence continues $\mathbb{H} \oplus \mathbb{H}$, $M(2, \mathbb{H}), M(4, \mathbb{C}), M(8, \mathbb{R}), M(8, \mathbb{R}) \oplus M(8, \mathbb{R}), M(16, \mathbb{R})$, and so on.

Let $\left(j_{1}, \ldots, j_{n}\right)$ be an orthonormal basis of $V$. Then $j_{k}$ are elements of $C_{n}$ and satisfy $j_{k}^{2}=-1$ and $j_{k} j_{l}=-j_{l} j_{k}$ for all $k, l=1, \ldots, n$ with $k \neq l$. So putting $A=C_{n}$ and $B=\left\{j_{1}, \ldots, j_{n}\right\}$, we see that any $A$-module gives rise to a geometric structure consisting of $n$ anticommuting complex structures, and conversely, any such geometric structure comes from an $A$ - module. In $[\mathbf{1}, \S 5]$, modules over Clifford algebras are classified, and this gives all possible geometric structures composed of anticommuting complex structures.

In $\S 2$ we define some notation and use it to put the integrability condition for a complex structure in a simple form. Then $\S 3$ gives a construction for the structures of Definition 1.1, using biquotients $C \backslash D / E$ of Lie groups. Sections 4 and 5 apply this construction in different ways, to produce in particular geometric structures that are not locally homogeneous, and compact manifolds with nontrivial geometric structures. In $\S 6$ we shall consider complex manifolds with affine connections and show that if the curvature of the connection satisfies a condition related to the Kähler structure, then the tangent and cotangent bundles of the manifold admit a geometric structure composed of two commuting complex structures. This may be regarded as a sort of Penrose transform. The results are applied to hypercomplex manifolds in $\S 7$.

\section{The complex decomposition of tensors}

Let $X$ be an almost complex manifold, with almost complex structure $J$, which will be written with indices as $J_{k}^{l}$ with respect to some real coordinate system $\left(x^{1}, \ldots, x^{2 n}\right)$. Let $S=S_{\ldots}^{a \ldots}$ be a tensor on $X$, taking values in $\mathbb{C}$. Here $a$ is a contravariant index of $S$, and any other indices of $S$ are represented by dots. The Greek characters $\alpha, \beta, \gamma, \delta, \epsilon$, and the starred characters $\alpha^{*}, \beta^{*}, \gamma^{*}, \delta^{*}, \epsilon^{*}$, will be used in place of the Roman indices $a, b, c, d, e$ respectively. They are tensor indices with respect to $\left(x^{1}, \ldots, x^{2 n}\right)$ in the normal sense, and their use is actually a shorthand indicating a modification to the tensor itself.

Define $S_{\ldots}^{\alpha \ldots}=\left(S_{\ldots}^{a \ldots}-i J_{k}^{a} S_{\ldots}^{k \ldots}\right) / 2$ and $S_{\ldots}^{\alpha^{*} \cdots}=\left(S_{\ldots}^{a \ldots}+i J_{k}^{a} S_{\ldots}^{k \ldots}\right) / 2$. In the same way, if $b$ is a covariant index on a complex-valued tensor $T_{b \ldots}^{\ldots}$, define $T_{\ddot{\beta} \ldots}^{\ldots}=\left(T_{\ddot{b} \ldots}^{\ldots}-i J_{b}^{k} T_{k \ldots} \ldots\right) / 2$ and $T_{\beta^{*} \ldots}=\left(T_{b \ldots}^{\ldots}+i J_{b}^{k} T_{k_{\ldots} \ldots}\right) / 2$. Then $S_{\ldots}^{\alpha \ldots}$ and $T_{\beta_{\ldots} \ldots}$ are the components of $S$ and $T$ that are complex linear w.r.t. $J$, and the starred versions are the components that are complex antilinear w.r.t. $J$. These operations are projections, and satisfy $S_{\cdots}^{a \cdots}=S_{\cdots}^{\alpha \ldots}+S_{\cdots}^{\alpha^{*} \cdots}$ and $T_{\ddot{b} \ldots}=T_{\ddot{\beta} \ldots}+T_{\ddot{\beta}^{*} \ldots}$. The complex decomposition of a real-valued tensor is self-adjoint. This means that changing round starred and unstarred indices has the same effect as complex conjugation.

The integrability conditions for the almost complex structure can be written conve- 
niently in this form. For $u, v$ vector fields, write $N_{J}(u, v)=[u, v]+J([J u, v]+[u, J v])-$ $[J u, J v]$. Then $N_{J}$ is called the Nijenhuis tensor of $J$, and it can easily be shown that $N_{J}$ depends only on the pointwise values of $u, v$, and not on their derivatives, so that $N_{J}$ is a true tensor. The Newlander-Nirenberg Theorem states that $J$ is an integrable complex structure if and only if $N_{J}=0$.

In our notation, we give $u=u^{a}$ and $v=v^{b}$ raised indices to indicate that they are vectors, and then the definitions give $u^{\alpha}=(u-i J u) / 2$ and $v^{\beta}=(v-i J v) / 2$. This gives

$$
\left[u^{\alpha}, v^{\beta}\right]=\frac{1}{4}[u-i J u, v-i J v]=\frac{1}{4}\{[u, v]-[J u, J v]-i([J u, v]+[u, J v])\}=w^{c},
$$

say. But then by definition, $w^{\gamma^{*}}=\frac{1}{8}\left(N_{J}(u, v)+i J N_{J}(u, v)\right)$. Thus the Nijenhuis tensor vanishes if $w^{\gamma^{*}}=0$ for all $u, v$, and so the integrability condition for $J$ is that $\left[u^{\alpha}, v^{\beta}\right]=w^{\gamma}$, that is, for every $u^{\alpha}, v^{\beta}$, the commutator $\left[u^{\alpha}, v^{\beta}\right]$ should be of the form $w^{\gamma}$. Moreover, it is sufficient that this should hold for $u^{\alpha}, v^{\beta}$ in some finite-dimensional vector space of complex vector fields, such that at every point of $X$ the vector fields span the whole complexified tangent space.

\section{Constructing geometric structures using Lie groups}

Let $D$ be a Lie group, and let $C, E$ be Lie subgroups of $D$, possibly discrete. Then $C \backslash D / E$ is the double coset space of double cosets $C d E$ for $d \in D$. We shall refer to $C \backslash D / E$ as a biquotient. Since $C, D$ and $E$ are Lie groups, the biquotient $C \backslash D / E$ has the structure of a manifold with singularities, so that some open set of $C \backslash D / E$ is a manifold.

Let $A$ be an algebra and $B \subset\left\{a \in A: a^{2}=-1\right\}$. We will shortly explain how to use data on the Lie algebras $\mathfrak{c}, \mathfrak{d}$ and $\mathfrak{e}$ of $C, D$ and $E$ to define an $A$-module structure on each tangent space of an open subset $C \backslash U / E$ of the biquotient $C \backslash D / E$. Thus each element of $B$ gives rise to an almost complex structure on this subset. We shall show that under certain conditions each of these almost complex structures is integrable, so that they form a geometric structure associated to $B$, as in $\S 1$. This gives a general construction for geometric structures of this form, which will be applied in the following sections. The result is loosely based on $[\mathbf{7}, \S 4]$.

Let $\mathfrak{d}=\mathfrak{c} \oplus \mathfrak{S}$, where $\mathfrak{S}$ is a vector subspace (not necessarily a Lie subalgebra) of $\mathfrak{d}$ containing $\mathfrak{e}$, that is invariant as a subspace under the adjoint action ad $(e)$ of all $e \in E$. The tangent space of $D / E$ at $E$ is $\mathfrak{d} / \mathfrak{e}$, which contains $\mathfrak{S} / \mathfrak{e}$ as a subspace. Because of the invariance of $\mathfrak{S}$ by $E$, this is the fibre at $E$ of a subbundle $S$ of $T(D / E)$ that is preserved by the left action of $D$ on $D / E$. Define the open set $U$ of $D$ by

$$
U=\left\{u \in D: u^{-1} C u \cap E=\{1\} \quad \text { and } \quad \mathfrak{c} \cap \operatorname{ad}(u) \mathfrak{S}=\{0\}\right\} .
$$

Here the first condition ensures that $C u E$ is a manifold point of $C \backslash D / E$, and the second implies that at $u E$, the fibre of $S$ is transverse to the left action of $C$, so that the projection from $U / E$ to $C \backslash U / E$ induces an isomorphism between the fibres of $S$ and the tangent spaces of $C \backslash U / E$.

Now let $\mathfrak{S} / \mathfrak{e}$ be given an $A$ - module structure that is invariant under the adjoint action of $E$. Then the fibres of $S$ have a left-invariant $A$ - module structure, and projecting to 
$C \backslash U / E$ defines an $A$ - module structure on its tangent bundle by the isomorphism above. Therefore $C \backslash U / E$ is a manifold, with an $A$ - module structure on each tangent space.

TheOREM 3.1. In the situation above, let $j \in B$ and define $V \subset \mathfrak{d} \otimes \mathbb{C}$ by

$$
V=\{u-i v: u \in \mathfrak{S} \quad \text { and } \quad v \in j \cdot(u+\mathfrak{e})\} .
$$

If $[V, V] \subset V$ in the Lie algebra $\mathfrak{d} \otimes \mathbb{C}$, then the almost complex structure $J$ defined by $j$ on $C \backslash U / E$ is integrable. Thus if this holds for all $j \in B$, there is a geometric structure on $C \backslash U / E$ associated to $B$.

Proof. For the proof we work on $C \backslash U$, and then project to $C \backslash U / E$. The reason for this is the interplay between left and right group actions: the set of left-invariant vector fields on $D$ is exactly the set of vector fields generating the right action of $D$ on $D$. So to define left-invariant structures on $D$, we work in terms of the vector fields of the right action of $D$ on $D$, and these push down to $C \backslash D$ immediately. Let $\phi: \mathfrak{d} \otimes \mathbb{C} \rightarrow T(C \backslash U) \otimes \mathbb{C}$ be the complexification of the natural map associating to an element of $\mathfrak{d}$ the restriction of the corresponding vector field on $C \backslash D$ from the right action of $D$ on $C \backslash D$. Then by Lie theory, $\phi$ has the property that $[\phi(u), \phi(v)]=\phi([u, v])$, and by $(2),\left.\phi\right|_{\mathfrak{S}}$ gives an isomorphism between $\mathfrak{S}$ and each tangent space of $C \backslash U$.

Let $W$ be the subbundle of $T(C \backslash U) \otimes \mathbb{C}$ identified with $V$ by $\phi$ at each point. We claim that for any sections $x, y$ of $W$, the Lie bracket $[x, y]$ of complex vector fields is also a section of $W$. To prove this, let $v_{1}, \ldots, v_{n}$ be a basis of $V$ over $\mathbb{C}$. Then $x=\Sigma_{k} x_{k} \phi\left(v_{k}\right)$ and $y=\Sigma_{k} y_{k} \phi\left(v_{k}\right)$, where $x_{k}$ and $y_{k}$ are smooth complex functions on $C \backslash U$. Therefore

$$
[x, y]=\sum_{k, l=1}^{n}\left(x_{k} \nabla_{\phi\left(v_{k}\right)} y_{l}\right) \phi\left(v_{l}\right)-\left(y_{l} \nabla_{\phi\left(v_{l}\right)} x_{k}\right) \phi\left(v_{k}\right)+x_{k} y_{l} \cdot\left[\phi\left(v_{k}\right), \phi\left(v_{l}\right)\right]
$$

so it is sufficient to show that $\left[\phi\left(v_{k}\right), \phi\left(v_{l}\right)\right]$ is a section of $W$. But this is trivial because $\left[\phi\left(v_{k}\right), \phi\left(v_{l}\right)\right]=\phi\left(\left[v_{k}, v_{l}\right]\right)$ and $[V, V] \subset V$, so $[x, y] \in \Gamma(W)$.

Now from the definition of the $A$ - module structure on the tangent spaces of $C \backslash U / E$ and the remarks above about the relation between left-invariance and the vector fields $\phi(\mathfrak{d})$, it can be seen that the subbundle $W$ of $T(C \backslash U) \otimes \mathbb{C}$ is exactly the inverse image under the projection from $C \backslash U$ to $C \backslash U / E$ of the bundle of complex vectors of the form $(1-i J) v$. So let $u^{\alpha}, v^{\beta}$ be complex vector fields on $C \backslash U / E$ of this form, in the notation of $\S 2$. Then we can lift $u^{\alpha}, v^{\beta}$ to sections $x, y$ of $W$ on $C \backslash U$, and we may in addition require $x, y$ to be invariant under the right action of $E$.

As we showed above, $[x, y]$ is a section of $W$, which must also be $E$-invariant, and so projects down to a well-defined complex vector field $w$ on $C \backslash U / E$. This $w$ is of the form $w^{\gamma}$, as $W$ is the lift of vectors $(1-i J) v$. Since $x, y$ are lifts of $u^{\alpha}, v^{\beta}$ and $[x, y]$ pushes down to $w^{\gamma}$, for elementary reasons we have $\left[u^{\alpha}, v^{\beta}\right]=w^{\gamma}$. But this is the criterion for integrability of $J$ given in $\S 2$. Therefore $J$, the almost complex structure defined by $j$, is integrable.

If $C$ is discrete, then the structure on the biquotient $C \backslash U / E$ is locally homogeneous by the left action of $D$. There are two other cases when this happens. Firstly, if $C$ is a 
normal subgroup of $D$, then the resulting structure is a homogeneous structure on $Q / E$, where $Q$ is the quotient group $D / C$. (In a similar way, if $E$ is normal we can pass to quotient subgroups.) Secondly, if $\mathfrak{S}$ is a Lie subalgebra of $\mathfrak{d}$, then $\mathfrak{S}$ is the Lie algebra of a subgroup $Q$ of $D$, and there is a local isomorphism between $C \backslash U / E$ and $Q / E$ taking the structure on $C \backslash U / E$ to a left-invariant structure on $Q / E$. So for the geometric structure of Theorem 3.1 to be locally inhomogeneous, $\mathfrak{c}$ cannot be normal in $\mathfrak{d}$, and $\mathfrak{S}$ cannot be a Lie subalgebra of $\mathfrak{d}$.

\section{Examples}

Here are some examples of the construction of $\S 3$.

Example 1. In $[\mathbf{1 1}, \S 6]$ and later in $[\mathbf{7}, \S 4]$, many compact semisimple groups $D$ were shown to have homogeneous hypercomplex structures. We may therefore ask if there are homogeneous geometric structures associated to $B$ on compact, semisimple, nonabelian $D$ for other interesting $A, B$. For $A$ the Clifford algebra $C_{n}$ of $\S 1$ with $n>3$ and $B=$ $\left\{j_{1}, \ldots, j_{n}\right\}$, the answer is no. This has been proved by Spindel et al., as the main result of [11], which is a Physics paper about supersymmetric sigma-models. They also claim the result for noncompact groups $D$, but we shall shortly see that this is false. The problem comes on $[\mathbf{1 1}$, p. 676], the sentence after $(4.37)$, in which they reduce the noncompact case to the compact case, and is because of a confusion about the notion of a positive system of roots for the Lie algebra of a noncompact group.

The most obvious groups carrying many homogeneous complex structures are matrix groups. Let $A$ be an algebra, and let $B \subset\left\{a \in A: a^{2}=-1\right\}$. Then $M(n, A)$ is the algebra of $n \times n$ matrices with entries in $A$. Let $G L(n, A)$ be the subset of invertible elements of $M(n, A)$. Then $D=G L(n, A)$ is a Lie group under multiplication. As examples, we could take $A=G L(m, \mathbb{F})$ for $\mathbb{F}=\mathbb{R}, \mathbb{C}$ or $\mathbb{H}$, and then $G L(n, A)=G L(m n, \mathbb{F})$ is a semisimple, noncompact Lie group. Left multiplication by $A$ gives an $A$ - module structure upon $\mathfrak{d}=M(n, A)$. Putting $C=E=\{1\}$ and $\mathfrak{S}=\mathfrak{d}$, it is easy to verify that this $A$ - module structure satisfies the conditions of Theorem 3.1, and defines a homogeneous geometric structure on $D$.

For example, $A=M(2, \mathbb{H})$ is the Clifford algebra $C_{4}$, so the noncompact, semisimple Lie group $G L(2, \mathbb{H})$ carries four homogeneous, anticommuting complex structures, which is a counterexample to the claim made by Spindel et al. and mentioned above. Unfortunately, geometric structures produced in this way are always trivial, as they are induced from the $A$ - module structure on $M(n, A)$ by the inclusion $D \subset M(n, A)$. However, even these trivial structures are of interest to physicists working upon supersymmetric sigma-models. (See for example [11] and references therein.)

Example 2. Here is a construction using nilpotent Lie groups. In examples it usually yields nontrivial, inhomogeneous structures. Let $A$ be an algebra, and let $B \subset\{a \in A$ : $\left.a^{2}=-1\right\}$. Let $Y$ be a nonzero $A$ - module and $Z$ be a real vector space. Suppose that $p: Y \times Y \rightarrow Z$ is a nonzero, bilinear, antisymmetric map satisfying $p\left(y_{1}, y_{2}\right)=p\left(j \cdot y_{1}, j \cdot y_{2}\right)$ for $j \in B$ and $y_{1}, y_{2} \in Y$, and suppose that $r: Z \rightarrow Y$ is a nonzero linear map satisfying $p\left(r\left(z_{1}\right), r\left(z_{2}\right)\right)=0$ for $z_{1}, z_{2} \in Z$. Let $D=Y \oplus Z$. We define a group operation on $D$ by

$$
\left(y_{1}, z_{1}\right) \circ\left(y_{2}, z_{2}\right)=\left(y_{1}+y_{2}, z_{1}+z_{2}+p\left(y_{1}, y_{2}\right)\right)
$$


With this operation, $D$ is a nonabelian, nilpotent Lie group with identity $1=(0,0)$, and Lie algebra $\mathfrak{d}=Y \oplus Z$. Define $C, E \subset D$ by $C=\{(z, r(z)): z \in Z\}$, and $E=\{(0,0)\}$. Then by (5) and the condition $p\left(r\left(z_{1}\right), r\left(z_{2}\right)\right)=0, C$ and $E$ are abelian subgroups of $D$. Define $\mathfrak{S}=Y \subset \mathfrak{d}=Y \oplus Z$, and let the $A$-module structure on $\mathfrak{S} / \mathfrak{e}=\mathfrak{S}$ be the $A$ module structure of $Y$.

Lemma 4.1. With these definitions, Theorem 3.1 defines a geometric structure on $C \backslash U$ associated to $B$.

Proof. Let $j \in B$. Then the subspace $V \subset \mathfrak{d} \otimes \mathbb{C}$ defined in Theorem 3.1 is $V=$ $\{y-i j \cdot y: y \in Y\}$. We must show that $[V, V] \subset V$. Now $\left[(1-i j) \cdot y,(1-i j) \cdot y^{\prime}\right]=$ $2 p\left(y, y^{\prime}\right)-2 p\left(j \cdot y, j \cdot y^{\prime}\right)-2 i p\left(j \cdot y, y^{\prime}\right)-2 i p\left(y, j \cdot y^{\prime}\right)=0$, using (5) and the relation $p\left(y_{1}, y_{2}\right)=p\left(j \cdot y_{1}, j \cdot y_{2}\right)$. Therefore $[V, V]=\{0\}$, and as this holds for each $j \in B$, Theorem 3.1 applies to give a geometric structure on $C \backslash U$ associated to $B$.

Example 3. Let $D$ be the Lie group $S U(2) \times S U(1,1)$, and let the Lie algebra $\mathfrak{d}$ of $G$ have basis $\left(u_{1}, u_{2}, u_{3}, v_{1}, v_{2}, v_{3}\right)$, where $\left\langle u_{1}, u_{2}, u_{3}\right\rangle=\mathfrak{s u}(2)$ and $\left\langle v_{1}, v_{2}, v_{3}\right\rangle=\mathfrak{s u}(1,1)$, and the Lie algebra relations are given by

$$
\begin{array}{cc}
{\left[u_{1}, u_{2}\right]=u_{3}} & {\left[u_{2}, u_{3}\right]=u_{1}, \quad\left[u_{3}, u_{1}\right]=u_{2},} \\
{\left[v_{1}, v_{2}\right]=-v_{3},} & {\left[v_{2}, v_{3}\right]=v_{1}, \quad\left[v_{3}, v_{1}\right]=v_{2}, \quad\left[u_{k}, v_{l}\right]=0}
\end{array}
$$

Let $C$ and $E$ be the connected subgroups of $D$ with Lie algebras $\mathfrak{c}=\left\langle v_{3}\right\rangle$ and $\mathfrak{e}=\left\langle u_{3}+v_{3}\right\rangle$. Then $C, E$ are isomorphic to $U(1)$. Define $\mathfrak{S}$ by $\mathfrak{S}=\left\langle u_{1}, u_{2}, v_{1}, v_{2}, u_{3}+v_{3}\right\rangle$. Then $\mathfrak{d}=\mathfrak{c} \oplus \mathfrak{S}, \mathfrak{e} \subset \mathfrak{S}$, and $\mathfrak{S}$ is an $E$ - invariant subspace of $\mathfrak{d}$, as we require. Now $\mathfrak{S} / \mathfrak{e}$ has basis $u_{1}, u_{2}, v_{1}, v_{2}$. Define complex structures $j_{1}, j_{2}, j_{3}$ on $\mathfrak{S} / \mathfrak{e}$ by

$$
\begin{array}{cccc}
j_{1} \cdot u_{1}=u_{2}, & j_{1} \cdot u_{2}=-u_{1}, & j_{1} \cdot v_{1}=-v_{2}, & j_{1} \cdot v_{2}=v_{1}, \\
j_{2} \cdot u_{1}=v_{1}, & j_{2} \cdot u_{2}=v_{2}, & j_{2} \cdot v_{1}=-u_{1}, & j_{2} \cdot v_{2}=-u_{2},
\end{array}
$$

and $j_{3}=j_{1} j_{2}$. Then $j_{1}, j_{2}, j_{3}$ are invariant under the action of $E$ on $\mathfrak{S} / \mathfrak{e}$. Calculation shows that each of $j_{1}, j_{2}$ and $j_{3}$ satisfy the condition $[V, V] \subset V$ of Theorem 3.1. Therefore Theorem 3.1 shows that there is a hypercomplex structure on the noncompact 4-manifold $C \backslash U / E$. Since $C \subset S U(1,1)$, the subgroup $S U(2) \subset D$ commutes with $C$, and thus $S U(2)$ acts on the left on $C \backslash U / E$ preserving the hypercomplex structure. In fact, a computation in coordinates shows that we have constructed the Eguchi-Hanson space [5], which is a well-known explicit hypercomplex structure on the noncompact 4-manifold $T^{*} \mathbb{C P}^{1}$.

Example 4. Here is a generalization of Example 3. Let $G$ be a connected Lie group and $H$ a connected Lie subgroup of $G$, and suppose that $\mathfrak{g}=\mathfrak{h} \oplus \mathfrak{m}$, where $\mathfrak{m}$ is a vector subspace of $\mathfrak{g}$ that is invariant under the adjoint action of $H$. Suppose that $[\mathfrak{h}, \mathfrak{m}] \subset \mathfrak{m}$ and that $[\mathfrak{m}, \mathfrak{m}] \subset \mathfrak{h}$. Suppose that $j$ is a complex structure on $\mathfrak{m}$ that is invariant under the adjoint action of $H$, that satisfies $\left[m_{1}, m_{2}\right]=\left[j \cdot m_{1}, j \cdot m_{2}\right]$ for all $m_{1}, m_{2} \in \mathfrak{m}$. For the case of Example 3 we put $G=S U(2)$ and $H=U(1)$ with Lie algebra $\left\langle u_{3}\right\rangle, \mathfrak{m}=\left\langle u_{1}, u_{2}\right\rangle$, and $j \cdot u_{1}=u_{2}, j \cdot u_{2}=-u_{1}$. In general, if $G / H$ is a Kähler symmetric space then the above conditions will hold.

Let $\tilde{G}$ be the Lie group with Lie algebra $\tilde{\mathfrak{g}}=\tilde{\mathfrak{h}} \oplus \tilde{\mathfrak{m}}$, where $\tilde{\mathfrak{h}}$ and $\tilde{\mathfrak{m}}$ are isomorphic to $\mathfrak{h}$ and $\mathfrak{m}$ respectively as vector spaces, but the Lie bracket is given by 


$$
\left[\left(h_{1}, m_{1}\right),\left(h_{2}, m_{2}\right)\right]=\left(\left[h_{1}, h_{2}\right]-\left[m_{1}, m_{2}\right],\left[h_{1}, m_{2}\right]+\left[m_{1}, h_{2}\right]\right) .
$$

Note the sign of the $\left[m_{1}, m_{2}\right]$ term. Then $\tilde{\mathfrak{g}}$ is a Lie algebra, so that $\tilde{G}$ is a Lie group, and the subalgebra $\tilde{\mathfrak{h}}$ generates a connected subgroup $\tilde{H}$ of $\tilde{G}$ that is isomorphic to $H$, modulo quotients by discrete subgroups of the centres of $G, \tilde{G}$. Let $D=G \times \tilde{G}$, let $C=\tilde{H} \subset D$, and let $E$ be the 'diagonal subgroup' of $H \times \tilde{H} \subset D$, composed of elements of the form $(h, h)$ using the identification $H \cong \tilde{H}$. Define $\mathfrak{S}=\mathfrak{e} \oplus \mathfrak{m} \oplus \tilde{\mathfrak{m}}$. Then $\mathfrak{d}=\mathfrak{c} \oplus \mathfrak{S}, \mathfrak{e} \subset \mathfrak{S}, \mathfrak{S}$ is invariant under the adjoint action of $E$, and $\mathfrak{S} / \mathfrak{e} \cong \mathfrak{m} \oplus \tilde{\mathfrak{m}}$.

Define complex structures $j_{1}, j_{2}$ and $j_{3}$ on $\mathfrak{S} / \mathfrak{e}$ by

$$
j_{1} \cdot(m, \tilde{m})=(j \cdot m,-j \cdot \tilde{m}), \quad j_{2} \cdot(m, \tilde{m})=(\tilde{m},-m), \quad \text { and } \quad j_{3}=j_{1} j_{2} .
$$

Then $B=\left\{j_{1}, j_{2}, j_{3}\right\}$ is a hypercomplex structure on $\mathfrak{S} / \mathfrak{e}$. The complex structures are invariant under the adjoint action of $E$. It is easy to show that $j_{1}, j_{2}$ and $j_{3}$ satisfy the condition $[V, V] \subset V$ of Theorem 3.1. Therefore Theorem 3.1 shows that the biquotient $C \backslash U / E$ has a hypercomplex structure. Since $C \subset \tilde{G}$, the subgroup $G$ of $D$ commutes with $C$, and therefore the hypercomplex structure on $C \backslash U / E$ is invariant under the left action of $G$.

Thus we have shown that for each Kähler symmetric space $G / H$ of dimension $2 k$, there is an associated hypercomplex manifold $C \backslash U / E$ of dimension $4 k$ invariant under $G$, that in fact contains $G / H$ as a submanifold. This situation is very reminiscent of the hypercomplex structures constructed on some coadjoint orbits of a semisimple complex group by Kronheimer [8], [9], using instanton moduli spaces. It seems feasible that there is some general construction of hypercomplex structures on biquotients that will include all of Kronheimer's examples as special cases, but the author has not yet found such a construction.

\section{Compact examples}

Let $A$ be an algebra, and let $B \subset\left\{a \in A: a^{2}=-1\right\}$ contain $j^{\prime}, j^{\prime \prime}$ with $j^{\prime} \neq \pm j^{\prime \prime}$. Let $Y, Z$ be nonzero $A$ - modules, and $p: Y \times Y \rightarrow Z$ be a nonzero, bilinear, antisymmetric map satisfying $p\left(y_{1}, y_{2}\right)=p\left(j \cdot y_{1}, j \cdot y_{2}\right)$ for $j \in B$, such that for some $y^{\prime}, y^{\prime \prime} \in Y$ we have $j^{\prime} \cdot p\left(j^{\prime} \cdot y^{\prime}, y^{\prime \prime}\right) \neq j^{\prime \prime} \cdot p\left(j^{\prime \prime} \cdot y^{\prime}, y^{\prime \prime}\right)$. Let $D=Y \oplus Z$. As in Example 2 of $\S 4$, define group multiplication on $D$ by

$$
\left(y_{1}, z_{1}\right) \circ\left(y_{2}, z_{2}\right)=\left(y_{1}+y_{2}, z_{1}+z_{2}+p\left(y_{1}, y_{2}\right)\right) .
$$

With this operation, $D$ is a nonabelian, nilpotent Lie group with identity $1=(0,0)$. The Lie algebra $\mathfrak{d}$ of $D$ is $Y \oplus Z$, which is an $A$ - module. Define $E$ to be $\{1\}$, and $C$ to be any discrete subgroup of $D$.

Proposition 5.1. The A-module structure on $\mathfrak{d}$ induces a nontrivial geometric structure on $C \backslash D$ associated to $B$.

Proof. We shall apply Theorem 3.1. As $C, E$ are discrete, $\mathfrak{c}=\mathfrak{e}=\{0\}$, and so $\mathfrak{S}=\mathfrak{d}$ in the set-up of $\S 3$; also $U=D$ and for $j \in B, V=(1-i j) \cdot \mathfrak{d}$. To apply the 
theorem we must show that $[V, V] \subset V$. Let $u, v \in \mathfrak{d}$, with $Y$ - components $w, x$. Then $[(1-i j) \cdot u,(1-i j) \cdot v]=2 p(w, x)-2 p(j \cdot w, j \cdot x)-2 i p(j \cdot w, x)-2 i p(w, j \cdot x)=0$, using (10) and the relation $p(w, x)=p(j \cdot w, j \cdot x)$. Therefore $[V, V]=\{0\}$, and as this holds for each $j \in B$, Theorem 3.1 applies to give a geometric structure on $C \backslash D$ associated to $B$.

In the case $C=\{1\}$, we have a left-invariant geometric structure on $D$, and the case $C$ discrete is locally isomorphic to the case $C=\{1\}$. Now the structure can only be trivial if $D$ is acting on $\mathfrak{d}$ preserving the trivial geometric structure associated to $B$. It is easy to show that for $D$ of the simple form we have chosen, this happens exactly when

$$
p\left(y_{1}, y_{2}\right)=\phi\left(y_{1}\right) y_{2}-\phi\left(y_{2}\right) y_{1} \quad \text { for } y_{1}, y_{2} \in Y,
$$

where $\phi: Y \rightarrow \operatorname{Hom}_{B}(Y, Z)$ is a linear map, and $\operatorname{Hom}_{B}(Y, Z)$ is the set of all linear homomorphisms from $Y$ to $Z$ commuting with the action of each $j \in B$. Because $p\left(y_{1}, y_{2}\right)=$ $p\left(j \cdot y_{1}, j \cdot y_{2}\right)$ for $s \in S,(11)$ implies that $\phi$ satisfies $2 \phi\left(y_{1}\right) y_{2}=p\left(y_{1}, y_{2}\right)+j \cdot p\left(j \cdot y_{1}, y_{2}\right)$ for $y_{1}, y_{2} \in Y$. Thus such a map $\phi: Y \rightarrow \operatorname{Hom}_{B}(Y, Z)$ can exist only if $j_{1} \cdot p\left(j_{1} \cdot y_{1}, y_{2}\right)=$ $j_{2} \cdot p\left(j_{2} \cdot y_{1}, y_{2}\right)$ for all $y_{1}, y_{2} \in Y$ and $j_{1}, j_{2} \in B$. But in the definition of $p$, we required that $j^{\prime} \cdot p\left(j^{\prime} \cdot y^{\prime}, y^{\prime \prime}\right) \neq j^{\prime \prime} \cdot p\left(j^{\prime \prime} \cdot y^{\prime}, y^{\prime \prime}\right)$ for some $y^{\prime}, y^{\prime \prime} \in Y$ and $j^{\prime}, j^{\prime \prime} \in B$. Therefore no suitable map $\phi$ exists, and the geometric structure constructed above on $C \backslash D$ is nontrivial.

By choosing $C$ such that $C \backslash D$ is compact in Proposition 5.1, we will get a compact manifold with a nontrivial geometric structure associated to $B$. Let $A$ be an algebra, and let $B \subset\left\{a \in A: a^{2}=-1\right\}$ contain $j^{\prime}, j^{\prime \prime}$ with $j^{\prime} \neq \pm j^{\prime \prime}$. Suppose $T$ is an $A$ - module that admits at least one nonzero symmetric bilinear function $\chi: T \times T \rightarrow \mathbb{R}$ satisfying $\chi\left(t_{1}, t_{2}\right)=\chi\left(j \cdot t_{1}, j \cdot t_{2}\right)$ whenever $j \in B$ and $t_{1}, t_{2} \in T$.

Proposition 5.2. Let $A, B, T, j^{\prime}, j^{\prime \prime}$ and $\chi$ be as above. Then there exist compact, nontrivial torus bundles over tori that admit nontrivial geometric structures associated to $B$.

Proof. Using $\chi$ it is easy to find a nonzero, symmetric, bilinear map $q: T \times T \rightarrow T$ satisfying the conditions that $q\left(t_{1}, t_{2}\right)=q\left(j \cdot t_{1}, j \cdot t_{2}\right)$ for $j \in B$ and $t_{1}, t_{2} \in T$, that for some $\tau^{\prime}, \tau^{\prime \prime} \in T, j^{\prime} \cdot q\left(j^{\prime} \cdot \tau^{\prime}, \tau^{\prime \prime}\right) \neq j^{\prime \prime} \cdot q\left(j^{\prime \prime} \cdot \tau^{\prime}, \tau^{\prime \prime}\right)$, and that $\left\{q\left(t_{k}, t_{l}\right): k, l=1, \ldots, n\right\}$ generate a discrete subgroup of $T$ for some basis $\left\{t_{1}, \ldots, t_{n}\right\}$ of $T$ over $\mathbb{R}$. Let $U, V$ be finite-dimensional real vector spaces, and let $r: U \times U \rightarrow V$ be a nonzero, antisymmetric, bilinear map satisfying the condition that for some fixed basis $\left\{u_{1}, \ldots, u_{m}\right\}$ of $U$, the elements $\left\{r\left(u_{k}, u_{l}\right): k, l=1, \ldots, m\right\}$ generate a discrete subgroup of $V$. This can easily be arranged, for instance by taking $V=\Lambda^{2} U$.

Define $Y=T \otimes U$ and $Z=T \otimes V$, and $p: Y \times Y \rightarrow Z$ to be the bilinear map satisfying $p\left(t \otimes u, t^{\prime} \otimes u^{\prime}\right)=q\left(t, t^{\prime}\right) \otimes r\left(u, u^{\prime}\right)$. The $A$ - module structure on $T$ induces an $A$ module structure on $Y$ and $Z$, and $p$ is clearly nonzero and antisymmetric, and satisfies $p\left(y_{1}, y_{2}\right)=p\left(j \cdot y_{1}, j \cdot y_{2}\right)$ for $j \in B$ because $q$ does. Choose some $k, l$ such that $r\left(u_{k}, u_{l}\right) \neq 0$, and put $y^{\prime}=\tau^{\prime} \otimes u_{k}, y^{\prime \prime}=\tau^{\prime \prime} \otimes u_{l}$. Then $j^{\prime} \cdot p\left(j^{\prime} \cdot y^{\prime}, y^{\prime \prime}\right) \neq j^{\prime \prime} \cdot p\left(j^{\prime \prime} \cdot y^{\prime}, y^{\prime \prime}\right)$ as we require.

Now the definitions above ensure that that commutators of elements $t_{k} \otimes u_{l} \in Y \subset D$ generate a discrete subgroup of the abelian subgroup $Z$ of $D$. Choose a discrete lattice $\Lambda$ in $Z$ such that $2 \Lambda$ contains all these commutators, and $Z / \Lambda$ is a torus. Define

$$
C=\left\{\left(\Sigma_{k, l} n_{k l} t_{k} \otimes u_{l}, \lambda\right): n_{k l} \in \mathbb{Z}, \lambda \in \Lambda\right\}
$$


Then $C$ is a subgroup of $D$, since if $\left[t_{k} \otimes u_{l}, t_{k^{\prime}} \otimes u_{l^{\prime}}\right]=2 \lambda$, then $\left(t_{k} \otimes u_{l}\right) \circ\left(t_{k^{\prime}} \otimes u_{l^{\prime}}\right)=$ $t_{k} \otimes u_{l}+t_{k^{\prime}} \otimes u_{l^{\prime}}+\lambda$ by (10), so $C$ is closed under multiplication, and also has inverses. Moreover, $C \backslash D$ is compact, as it is easily shown to be a (nontrivial) torus bundle over a torus, where the fibre is $Z / \Lambda$ and the base space is $Y$ divided by the lattice generated by the elements $t_{k} \otimes u_{l}$. The definitions we have made satisfy all the necessary conditions, so Proposition 5.1 applies, and the proof is complete.

When $A$ is the Clifford algebra $C_{n}$ of $\S 1$ for $n>1$ and $B=\left\{j_{1}, \ldots, j_{n}\right\}$, we may take $T=A$ and $\chi\left(t, t^{\prime}\right)=\left\langle t, t^{\prime}\right\rangle$ using the natural inner product, and then Proposition 5.2 shows that there are nontrivial compact examples of manifolds with $n$ anticommuting complex structures. More generally, if $A$ is an algebra with a vector space automorphism $*$ satisfying $*^{2}=1$ and $a^{*} b^{*}=(b a)^{*}$ for $a, b \in A$, then suitable modules and functions $T, \chi$ also exist.

\section{Connections on complex manifolds}

Let $X$ be a complex manifold with complex structure $J$, and let $\nabla$ be a torsionfree connection on $X$ satisfying $\nabla J=0$, which will be written in the usual way as $\Gamma_{b c}^{a}$ relative to a local coordinate system $\left(x^{1}, \ldots, x^{2 n}\right)$. In these coordinates, $\Gamma$ may be decomposed into components relative to $J$ as in $\S 2$, but as $\Gamma$ is not a tensor the decomposition does depend on the coordinate system. To avoid the complications this raises, we shall restrict to coordinate systems $\left(x^{1}, \ldots, x^{2 n}\right)$ with the property that $J$ is constant in coordinates, i.e. $\partial J_{b}^{a} / \partial x^{c}=0$.

As $\nabla J=0$ we have $\Gamma_{b c}^{a}=\Gamma_{\beta c}^{\alpha}+\Gamma_{\beta^{*} c}^{\alpha^{*}}$, and as $\nabla$ is torsion-free $\Gamma_{b c}^{a}=\Gamma_{c b}^{a}$. Together these imply that $\Gamma_{b c}^{a}=\Gamma_{\beta \gamma}^{\alpha}+\Gamma_{\beta^{*} \gamma^{*}}^{\alpha^{*}}$. Now the curvature $R_{b c d}^{a}$ of $\nabla$ is given by $R_{b c d}^{a}=$ $\partial \Gamma_{b d}^{a} / \partial x^{c}-\partial \Gamma_{b c}^{a} / \partial x^{d}+\Gamma_{k c}^{a} \Gamma_{b d}^{k}-\Gamma_{k d}^{a} \Gamma_{b c}^{k}$. Substituting in for $\Gamma$ gives $R_{b c d}^{a}=R_{\beta c d}^{\alpha}+R^{\alpha^{*}}{ }_{\beta^{*} c d}$. Since $\nabla$ is torsion-free, $R$ satisfies the Bianchi identity $R_{b c d}^{a}+R_{c d b}^{a}+R_{d b c}^{a}=0$, and thus $R_{\beta \gamma^{*} \delta^{*}}^{\alpha}+R_{\gamma^{*} \delta^{*} \beta}^{\alpha}+R_{\delta^{*} \beta \gamma^{*}}^{\alpha}=0$. From above the last two terms are zero, and so $R^{\alpha}{ }_{\beta \gamma^{*} \delta^{*}}=0$, and similarly $R_{\beta^{*} \gamma \delta}^{\alpha^{*}}=0$. Therefore

$$
R_{b c d}^{a}=R_{\beta \gamma \delta}^{\alpha}+R_{\beta \gamma^{*} \delta}^{\alpha}+R_{\beta \gamma \delta^{*}}^{\alpha}+R_{\beta^{*} \gamma^{*} \delta^{*}}^{\alpha^{*}}+R_{\beta^{*} \gamma^{*} \delta}^{\alpha^{*}}+R_{\beta^{*} \gamma \delta^{*}}^{\alpha^{*}}
$$

Now by $[\mathbf{4}$, Lemma 5], the curvature tensor of a Kähler manifold satisfies

$$
R_{b c d}^{a}=R_{\beta \gamma^{*} \delta}^{\alpha}+R_{\beta \gamma \delta^{*}}^{\alpha}+R_{\beta^{*} \gamma^{*} \delta}^{\alpha^{*}}+R_{\beta^{*} \gamma \delta^{*}}^{\alpha^{*}}
$$

So the curvature of the Levi-Civita connection of a Kähler metric satisfies (14), whereas the curvature of a torsion-free $G L(n, \mathbb{C})$ - connection $\nabla$ only need satisfy (13), which is weaker. For $\nabla$ to satisfy (14) it is necessary and sufficient that it should satisfy the additional condition $R^{\alpha}{ }_{\beta \gamma \delta}=0$.

Definition 6.1. A torsion-free connection $\nabla$ on a complex manifold $(X, J)$ with $\nabla J=0$ is called complex-flat if the curvature $R$ of $\nabla$ satisfies (14), or equivalently if $R_{\beta \gamma \delta}^{\alpha}=0$.

The tangent bundle $T X$ of $X$ is naturally a complex manifold, with complex structure also denoted $J$. Its tangent space $T(T X)$ splits into a direct sum $H \oplus V$, where $H$ is the horizontal subspaces of the connection $\nabla$, and $V$ is the tangent spaces to the fibres of 
$T X \rightarrow X$. Now $V$ is closed under $J$ as $T X$ is a holomorphic bundle, and $H$ is closed under $J$ as $\nabla J=0$. Thus we may define an almost complex structure $K$ on $T X$, by $K=J$ on $H$ and $K=-J$ on $V$. Then $K$ commutes with $J$ and projects down to $J$ on $X$. In the Kähler case, the metric identifies $T X$ and $T^{*} X$, and $J, K$ are the complex structures on $T X$ and $T^{*} X$ respectively.

THEOREM 6.2. The almost complex structure $K$ is integrable if and only if $R_{\beta \gamma \delta}^{\alpha}=0$.

Proof. Let $\left(x^{1}, \ldots, x^{2 n}\right)$ be the local coordinates above, and let $\left(y^{1}, \ldots, y^{2 n}\right)$ be coordinates w.r.t. the basis $\left(\partial / \partial x^{1}, \ldots, \partial / \partial x^{2 n}\right)$ for the fibres of $T X$. Then $\left(x^{1}, \ldots, x^{2 n}\right.$, $\left.y^{1}, \ldots, y^{2 n}\right)$ are coordinates for $T X$. In the complex decomposition w.r.t. $J$, it is readily shown that

$$
K\left(p^{\alpha} \frac{\partial}{\partial x^{\alpha}}+q^{\alpha^{*}} \frac{\partial}{\partial y^{\alpha^{*}}}\right)=i p^{\alpha} \frac{\partial}{\partial x^{\alpha}}+i q^{\alpha^{*}} \frac{\partial}{\partial y^{\alpha^{*}}}-2 i \Gamma_{\beta \gamma}^{\alpha} y^{\beta} p^{\gamma} \frac{\partial}{\partial y^{\alpha}} .
$$

We deduce that a vector of the form $(1-i K) u$ may be written

$$
(1-i K) u=p^{\alpha} \frac{\partial}{\partial x^{\alpha}}+q^{\alpha^{*}} \frac{\partial}{\partial y^{\alpha^{*}}}-\Gamma_{\beta \gamma}^{\alpha} y^{\beta} p^{\gamma} \frac{\partial}{\partial y^{\alpha}} .
$$

Now from $\S 2$, we know that $K$ is integrable if and only if whenever $u, v$ are vector fields, there exists $w$ such that

$$
[(1-i K) u,(1-i K) v]=(1-i K) w,
$$

and it suffices for this to hold for $u, v$ in some finite-dimensional vector space of vector fields taking all possible values at each point. Therefore $K$ is integrable if and only if (17) holds for all complex vector fields $(1-i K) u,(1-i K) v$ of the form (16), where $p^{\alpha}$ and $q^{\alpha^{*}}$ are constant complex functions.

Defining $(1-i K) u$ by $(16)$ and $(1-i K) v$ by

$$
(1-i K) v=r^{\alpha} \frac{\partial}{\partial x^{\alpha}}+s^{\alpha^{*}} \frac{\partial}{\partial y^{\alpha^{*}}}-\Gamma_{\beta \gamma}^{\alpha} y^{\beta} r^{\gamma} \frac{\partial}{\partial y^{\alpha}}
$$

for constant functions $p^{\alpha}, q^{\alpha^{*}}, r^{\alpha}, s^{\alpha^{*}}$, we calculate

$$
\begin{aligned}
{[(1-i K) u,(1-i K) v]=} & r^{\delta} \frac{\partial \Gamma_{\beta \gamma}^{\alpha}}{\partial x^{\delta}} y^{\beta} p^{\gamma} \frac{\partial}{\partial y^{\alpha}}-p^{\gamma} \frac{\partial \Gamma_{\beta \delta}^{\alpha}}{\partial x^{\gamma}} y^{\beta} r^{\delta} \frac{\partial}{\partial y^{\alpha}} \\
& +\Gamma_{\beta \gamma}^{\epsilon} y^{\beta} p^{\gamma} \Gamma_{\epsilon \delta}^{\alpha} r^{\delta} \frac{\partial}{\partial y^{\alpha}}-\Gamma_{\beta \delta}^{\epsilon} y^{\beta} r^{\delta} \Gamma_{\epsilon \gamma}^{\alpha} p^{\gamma} \frac{\partial}{\partial y^{\alpha}} \\
= & -R_{\beta \gamma \delta}^{\alpha} y^{\beta} p^{\gamma} r^{\delta} \frac{\partial}{\partial y^{\alpha}},
\end{aligned}
$$

by the expression for $R_{b c d}^{a}$ given above. But from (16), no sum of the complex vectors $\partial / \partial y^{\alpha}$ can be of the form $(1-i K) w$, so (17) holds if and only if $R_{\beta \gamma \delta}^{\alpha} y^{\beta} p^{\gamma} r^{\delta}=0$ identically. Therefore the condition for $K$ to be integrable is that $R_{\beta \gamma \delta}^{\alpha}=0$. 
We may interpret Theorem 6.2 as a sort of miniature twistor transform, since a curvature condition translating to the integrability of an almost complex structure on an auxiliary bundle is exactly the same set-up as the Penrose transform for self-dual conformal 4-manifolds [2]. The structure on $T X$ is a geometric structure associated to $\{J \oplus J, J \oplus-J\}$.

The simplest examples of complex-flat manifolds are Kähler manifolds, taking $\nabla$ to be the Levi-Civita connection of the Kähler metric. However, there are ways of finding complex-flat manifolds with no compatible Kähler metric. For example, a complex-flat manifold $M$ can appear as a complex submanifold of another complex-flat manifold $X$; to induce a complex-flat connection on the submanifold one must choose a splitting of $\left.T X\right|_{M}$ into $T M$ and some other bundle holomorphic w.r.t. $K$. There is also a quotient for complex-flat manifolds based upon the ideas of $[\mathbf{6}]$; the moment map $\mu$ must satisfy the condition that $d \mu$ is a holomorphic section of $T^{*} X$ w.r.t. $K$.

Lemma 6.3. Let $X, J$ and $\nabla$ be as above. Then complex structures $J, K$ are defined on $T^{*} M$ as on $T M$. Let $\omega$ be the natural symplectic form on $T^{*} M$, and let $g(u, v)=$ $\omega(J K u, v)$ for vectors $u, v$. Then $g$ is a pseudo-riemannian metric, which is pseudo-Kähler w.r.t. $K$.

Proof. The tangent bundle $T\left(T^{*} X\right)$ splits as $H \oplus V$ as above, and by definition of $\omega,\left.\omega\right|_{H}=\left.\omega\right|_{V}=0$, so that $\omega$ is a section of $H^{*} \otimes V^{*}$. Since $J K=-1$ on $H$ and $J K=1$ on $V$, it follows that $\omega(J K u, v)=\omega(J K v, u)$, and $g$ is a pseudo-riemannian metric. Also $\omega(K u, v)=-\omega(u, K v)$, so $g(u, v)=g(K u, K v)$ and $g$ is pseudo-hermitian w.r.t. $K$. Define $\omega^{\prime}(u, v)=g(K u, v)$. Then $\omega^{\prime}(u, v)=-\omega(J u, v)$. But this is a closed form, as it is the imaginary part of the canonical holomorphic 2-form on $T^{*} M$, regarded as a complex manifold w.r.t. $J$. Thus $K$ is integrable, $g$ is pseudo-hermitian w.r.t. $K$, and the associated 2 -form is closed, so $g$ is pseudo-Kähler w.r.t. $K$.

\section{Hypercomplex structures and complex-flat structures}

Let $M$ be a hypercomplex manifold $(\S 1)$ with complex structures $J_{1}, J_{2}, J_{3}$. By [10, Proposition 9.12], there is a unique connection $\nabla$ on $M$ called the Obata connection, that is torsion-free and satisfies $\nabla J_{k}=0$. We shall show that $\nabla$ is a complex-flat connection for each of the complex structures $J_{k}$.

Proposition 7.1. Let $M, J_{k}$ and $\nabla$ be as above. Then the curvature $R_{b c d}^{a}$ of $\nabla$ satisfies $R_{\beta \gamma \delta}^{\alpha}=0$ in the complex decomposition w.r.t. each $J_{k}$. Thus $\nabla$ is a complex-flat connection w.r.t. each $J_{k}$.

Proof. We shall prove the result for $J_{1}$, for by symmetry it then holds for $J_{2}, J_{3}$. As $\nabla$ is torsion-free and $\nabla J_{k}=0$, from $\S 6$ the curvature $R$ satisfies $R_{b c d}^{a}=R_{\beta c d}^{\alpha}+$ $R^{\alpha^{*}}{ }_{\beta^{*} c d}$ in the complex decomposition w.r.t. $J_{k}$. Thus $\left(J_{2}\right)_{e}^{a} R_{b c d}^{e}=\left(J_{2}\right)_{b}^{e} R_{e c d}^{a}$. But as $J_{1} J_{2}=-J_{2} J_{1},\left(J_{2}\right)_{e}^{a}=\left(J_{2}\right)_{\epsilon_{*}^{*}}^{\alpha}+\left(J_{2}\right)_{\epsilon}^{\alpha^{*}}$ in the complex decomposition w.r.t. $J_{1}$. Therefore $\left(J_{2}\right)_{\epsilon}^{\alpha^{*}} R_{\beta \gamma \delta}^{\epsilon}=\left(J_{2}\right)_{\beta}^{\epsilon^{*}} R_{\epsilon^{*} \gamma \delta}^{\alpha^{*}}$ in the complex decomposition w.r.t. $J_{1}$. But from $\S 6$, $R^{\alpha^{*} \gamma \delta}=0$ so that the r.h.s. of this equation vanishes, and thus $R^{\alpha}{ }_{\beta \gamma \delta}=0$.

Under the natural identification $\mathbb{H}^{n} \cong \mathbb{R}^{4 n}$, define $B=\left\{a J_{1}+b J_{2}+c J_{3}: a^{2}+b^{2}+\right.$ $\left.c^{2}=1\right\} \subset G L(4 n)$. Then the geometric structure associated to $B$ is the $4 n$ - dimensional hypercomplex structure. Under the natural identification $\mathbb{H}^{n} \oplus \mathbb{H}^{n} \cong \mathbb{R}^{8 n}$, define $B^{\prime}=$ 
$\left\{j \oplus j^{\prime}: j, j^{\prime} \in B\right\} \subset G L(8 n)$. The subgroup of $G L(8 n)$ commuting with each element of $B^{\prime}$ is $G=G L(n, \mathbb{H}) \oplus G L(n, \mathbb{H})$.

Proposition 7.2. Let $M$ be a hypercomplex manifold of dimension $4 n$. Then TM and $T^{*} M$ admit natural geometric structures associated to $B^{\prime}$.

Proof. Let $N$ be $T M$ or $T^{*} M$, and write $T N=H \oplus V$, where $H$ is the horizontal subspaces of the Obata connection, and $V$ is the tangent spaces to the fibres of $N \rightarrow M$. Then $J_{1}, J_{2}, J_{3}$ are defined naturally on $N$, and $H$ and $V$ are closed under $J_{k}$. Thus we may write $J_{k}=\left.\left.J_{k}\right|_{H} \oplus J_{k}\right|_{V}$. The splitting $T N=H \oplus V$ and the actions of $J_{k}$ clearly give $T N$ a $G L(n, \mathbb{H}) \oplus G L(n, \mathbb{H})$ - structure, which therefore defines an almost complex structure on $N$ for each element of $B^{\prime}$. It remains to show that each of these structures is integrable. This can easily be deduced from the facts that $\left.\left.J_{k}\right|_{H} \oplus J_{k}\right|_{V}$ is integrable (from above), and $\left.J_{k}\right|_{H} \oplus-\left.J_{k}\right|_{V}$ is integrable by Theorem 6.2 .

Tensor powers of $T M, T^{*} M$ also have natural geometric structures in a similar way.

LEMma 7.3. Suppose that TM or $T^{*} M$ has a torsion-free connection $\nabla^{\prime}$ on its total space preserving the geometric structure of Proposition 7.2. Then the hypercomplex structure of $M$ is trivial.

Proof. As $\nabla^{\prime}$ preserves the complex structures, it preserves the $G L(n, \mathbb{H}) \oplus G L(n, \mathbb{H})$ structure and therefore the distribution of horizontal subspaces of the Obata connection $\nabla$ of $M$. But it can easily be shown using the Frobenius theorem that any subbundle of $T M$ preserved by a torsion-free $\nabla$ must be an integrable subbundle. Thus the Obata connection of $M$ is flat, and so the hypercomplex structure of $M$ is trivial.

Here is a construction of the Obata connection of a hypercomplex manifold:

Observation 7.4. Let $M, J_{k}$ and $\nabla$ be as above. Then $J_{k}$ induces a natural complex structure $J_{k}^{\prime}$ on $T^{*} M$. The horizontal subspaces of $\nabla$ are the kernels of $1+J_{1}^{\prime} J_{2}^{\prime} J_{3}^{\prime}$; this characterizes the Obata connection $\nabla$. Also, $T^{*} M$ has a natural pseudo-hyperkähler structure, following Lemma 6.3.

The theory of holonomy $([\mathbf{3}],[\mathbf{1 0}])$ is a way of providing a unified treatment for a wide class of geometric structures. The geometric structures it studies consist of a $G$ - structure on a manifold ( $\S 1)$, preserved by a torsion-free connection $\nabla$; the existence of $\nabla$ implies a partial differential equation on the $G$ - structure. Calculating with representation theory one can show that comparatively few groups $G$ lead to nontrivial structures.

From Lemma 7.3 we see that geometric structures associated to $B^{\prime}$ lie outside the theory of holonomy. In fact geometric structures fall into three classes according to the behaviour of the 1-jet of the structure at a point. Firstly, the 1-jet may always be uniquely isomorphic to the flat model; secondly, it may be isomorphic to the flat model, but in many ways; and thirdly, the 1-jet may not always be isomorphic to the flat model. In the first two cases the structure is preserved by a torsion-free connection, which is only unique in the first case. In the third case, which includes geometric structures associated to $B^{\prime}$, there need be no such connection. The 'curvature' of such a structure is visible at the level of 1 -jets.

Acknowledgements 
I would like to thank Kristoff Galicki for bringing reference [11] to my attention, and to thank Christ Church, Oxford and the Institute for Advanced Study, Princeton for hospitality. This work was partially supported by NSF grant no. DMS 9304580.

\section{REFERENCES}

1. M.F. Atiyah, R. Bott and A. Shapiro, 'Clifford Modules Part I', Topology 3 sup. 1 (1964), 3-38.

2. M.F. Atiyah, N.J. Hitchin and I.M. Singer, 'Self-duality in four-dimensional Riemannian geometry', Proc. Roy. Soc. Lond. A362 (1978), 425-461.

3. M. Berger, 'Sur les groupes d'holonomie homogène des variétés à connexion affines et des variétés riemanniennes', Bull. Soc. Math. France 83 (1955), 279-330.

4. S. Bochner, 'Vector fields and Ricci curvature', Bull. Amer. Math. Soc. 52 (1946), 776-797.

5. T. Eguchi and A.J. Hanson, 'Asymptotically flat solutions to Euclidean gravity', Phys. Lett. 74B (1991), 249-251.

6. D. Joyce, 'The hypercomplex quotient and the quaternionic quotient', Math. Ann. 290 (1991), 323-340.

7. D. Joyce, 'Compact hypercomplex and quaternionic manifolds', J. Diff. Geom. 35 (1992), 743-761.

8. P.B. Kronheimer, 'Instantons and the geometry of the nilpotent variety', J. Diff. Geom. 32 (1990), 473-490.

9. P.B. Kronheimer, 'A hyper-Kählerian structure on coadjoint orbits of a semisimple complex group', J. London Math. Soc. 42 (1990), 193-208.

10. S.M. Salamon, 'Riemannian geometry and holonomy groups', Pitman Res. Notes Math. Ser. 201 (1989), Longman Sci. Tech., Harlow.

11. Ph. Spindel, A. Sevrin, W. Troost and A. Van Proeyen, 'Extended super-symmetric $\sigma$ - models on group manifolds', Nucl. Phys. B308 (1988), 662-698.

Mathematical Institute

24-29 St. Giles'

Oxford

$O X 13 L B$ 